\title{
A Splitting Result for Real Submanifolds of a Kähler Manifold
}

\author{
Leonardo Biliotti ${ }^{1}$ (D)
}

Received: 24 January 2021 / Accepted: 2 October 2021 / Published online: 16 October 2021

(C) The Author(s) 2021

\begin{abstract}
Let $(Z, \omega)$ be a connected Kähler manifold with an holomorphic action of the complex reductive Lie group $U^{\mathbb{C}}$, where $U$ is a compact connected Lie group acting in a hamiltonian fashion. Let $G$ be a closed compatible Lie group of $U^{\mathbb{C}}$ and let $M$ be a $G$-invariant connected submanifold of $Z$. Let $x \in M$. If $G$ is a real form of $U^{\mathbb{C}}$, we investigate conditions such that $G \cdot x$ compact implies $U^{\mathbb{C}} \cdot x$ is compact as well. The vice-versa is also investigated. We also characterize $G$-invariant real submanifolds such that the norm-square of the gradient map is constant. As an application, we prove a splitting result for real connected submanifolds of $(Z, \omega)$ generalizing a result proved in Gori and Podestà (Ann Global Anal Geom 26: 315-318, 2004), see also Bedulli and Gori (Results Math 47: 194-198, 2005), Biliotti (Bull Belg Math Soc Simon Stevin 16: 107-116 2009).
\end{abstract}

Keywords Gradient map · Real reductive Lie groups · Carton decomposition

Mathematics Subject Classification 22E45 $\cdot 53 \mathrm{D} 20$

\section{Introduction}

Let $(Z, \omega)$ be a Kähler manifold. Assume that $U^{\mathbb{C}}$ acts holomorphically on $Z$, that $U$ preserves $\omega$ and that there is a momentum map for the $U$ action on $Z$. This means there is a map $\mu: Z \longrightarrow \mathfrak{u}^{*}$, where $\mathfrak{u}$ is the Lie algebra of $U$ and $\mathfrak{u}^{*}$ is its dual, which is $U$-equivariant with respect to the given action of $U$ on $Z$ and the coadjoint action $\mathrm{Ad}^{*}$ of $U$ on $\mathfrak{u}^{*}$ and satisfying the following condition. Let $\xi \in \mathfrak{u}$. We denote

The author was partially supported by the Project PRIN 2017 "Real and Complex Manifolds: Topology, Geometry and holomorphic dynamics" and by GNSAGA INdAM.

$凶$ Leonardo Biliotti

leonardo.biliotti@unipr.it

1 Leonardo Biliotti Dipartimento di Scienze Matematiche, Fisiche e Informatiche, Università di Parma, Parma, Italy 
by $\xi_{Z}$ the induced vector field on $Z$, i.e., $\xi_{Z}(p)=\left.\frac{\mathrm{d}}{\mathrm{dt}}\right|_{t=0} \exp (t \xi) p$. Let $\mu^{\xi}$ be the function $\mu^{\xi}(z):=\mu(z)(\xi)$, i.e., the contraction of the momentum map along $\xi$. Then $\mathrm{d} \mu^{\xi}=i_{\xi_{Z}} \omega$.

Let $G$ be a closed connected subgroup of $U^{\mathbb{C}}$ compatible with respect to the Cartan decomposition of $U^{\mathbb{C}}$, i.e. $G=K \exp (\mathfrak{p})$, for $K=U \cap G$ and $\mathfrak{p}=\mathfrak{g} \cap i \mathfrak{u}$ ( Helgason 2001; Knapp 2002). The inclusion $i \mathfrak{p} \hookrightarrow \mathfrak{u}$ induces by restriction a $K$-equivariant map $\mu_{i \mathfrak{p}}: Z \longrightarrow(i \mathfrak{p})^{*}$ (Heinzner et al. 2008; Heinzner and Stötzel 2006).

Let $\langle\cdot, \cdot\rangle$ be a $U$-invariant scalar product on $u$. Let $\langle\cdot, \cdot\rangle$ denote also the inner product on $i \mathfrak{u}$ such that multiplication by $i$ be an isometry of $\mathfrak{u}$ into $i \mathfrak{u}$. Hence we may identify $\mathfrak{u}^{*}$ and $\mathfrak{u}$ by means of $\langle\cdot, \cdot\rangle$ and so we view $\mu$ as a map $\mu: Z \longrightarrow \mathfrak{u}$. Therefore, we may view $\mu_{i \mathfrak{p}}$ as a map $\mu_{\mathfrak{p}}: Z \longrightarrow \mathfrak{p}$ as follows:

$$
\left\langle\mu_{\mathfrak{p}}(x), \beta\right\rangle=-\langle\mu(x), i \beta\rangle, \forall \beta \in \mathfrak{p} .
$$

We call $\mu_{\mathfrak{p}}$ the $G$-gradient map associated with $\mu$. We also set $\mu_{\mathfrak{p}}^{\beta}:=\left\langle\mu_{\mathfrak{p}}, \beta\right\rangle$. By definition, it follows that $\operatorname{grad} \mu_{\mathfrak{p}}^{\beta}=\beta_{Z}$. If $M$ is a $G$-stable locally closed real submanifold of $Z$, we may consider $\mu_{\mathfrak{p}}$ as a mapping $\mu_{\mathfrak{p}}: M \longrightarrow \mathfrak{p}$ such that $\operatorname{grad} \mu_{\mathfrak{p}}=\beta_{M}$, where the gradient is computed with respect to the induced Riemannian metric on $M$. Since $M$ is $G$-stable it follows $\beta_{Z}(p)=\beta_{M}(p)$ for any $p \in M$.

Assume that $G$ is a real form of $U^{\mathbb{C}}$. If $U^{\mathbb{C}} \cdot x$ is compact, then it is well-known that $G$ has a closed orbit contained in $U^{\mathbb{C}} \cdot x$ (Heinzner et al. 2008). On the other hand, if $G \cdot x$ is closed then it is not in general true that $U^{\mathbb{C}} \cdot x$ is closed as well (Guivarc'h et al. 1998). In Sect. 2, we investigate conditions such that $G \cdot x$ compact implies $U^{\mathbb{C}} \cdot x$ is compact. If $G \cdot x$ is compact then we give a necessary condition for $U^{\mathbb{C}} \cdot x$ to be compact. If $M$ is Lagrangian and $x \in M$, then $U^{\mathbb{C}} \cdot x$ being compact implies $G \cdot x$ is a Lagrangian submanifold of $U^{\mathbb{C}} \cdot x$. Finally, we study the case when $Z$ is $U^{\mathbb{C}}$-semistable, $M$ is $G$-semistable and is contained in the zero level set of the gradient map of $K^{\mathbb{C}}$. As an application we obtain a well-known result of Birkes (Birkes 1971).

A strategy for analyzing the $G$-action on $M$ is to view the function $v_{\mathfrak{p}}: M \longrightarrow \mathbb{R}$,

$$
v_{\mathfrak{p}}(x)=\left\|\mu_{\mathfrak{p}}(x)\right\|^{2}
$$

as a Morse-like function. The function $v_{\mathfrak{p}}$ is called the norm-square of the gradient map. If $M$ is compact or $\mu_{\mathfrak{p}}$ is proper, then associated to the critical points of $\nu_{\mathfrak{p}}$ we have $G$-stable submanifold of $M$ that they are strata of a Morse type stratification of $M$ (Heinzner et al. 2008; Kirwan 1984). In Sect. 3, we investigate under which condition $v_{\mathfrak{p}}$ is constant. The following result has some interest by itself.

Proposition 1 Let $M$ be a $G$-stable connected submanifold of $Z$ and let $\mu_{\mathfrak{p}}: M \longrightarrow \mathfrak{p}$ be the restricted gradient map. Then the norm-square of the gradient map $\nu_{\mathfrak{p}}: M \longrightarrow$ $\mathbb{R}$ is constant if and only if any $G$-orbit is compact.

By the stratification Theorem (Heinzner et al. 2008), it follows that $M$ coincides with a maximal pre-stratum and $\mu_{\mathfrak{p}}(M)=K \cdot \beta$. Moreover, $M=K \times_{K^{\beta}} \mu_{\mathfrak{p}}^{-1}(\beta)$, where $K^{\beta}=\{k \in K: \operatorname{Ad}(k)(\beta)=\beta\}$. Let $x \in \mu_{\mathfrak{p}}^{-1}(\beta)$. By the $K$-equivariance of $\mu_{\mathfrak{p}}$, 
it follows that the stabilizer $K_{x} \subseteq K^{\beta}$. Although $G \cdot x$ is compact, it is not true in general $K_{x}=K^{\beta}$. Indeed, let $U$ be a connected, compact semisimple Lie group and let $\rho: U \longrightarrow \mathrm{SL}(W)$ be a complex representation. Let $G$ be a noncompact connected semisimple real form of $U^{\mathbb{C}}$. It is well known that $U^{\mathbb{C}}$ has a compact orbit in $\mathbb{P}(W)$, which is a complex $U$-orbit (Guillemin and Sternberg 1990). Let $\mathcal{O}$ denote a compact orbit of $U^{\mathbb{C}}$. If $x \in \mathcal{O}$ realizes the maximum of the norm-square of the $G$-gradient map restricted to $\mathcal{O}$, then $G \cdot x$ is closed and it is a $K$-orbit (Heinzner et al. 2008). Now, $K_{x}=K \cap U^{\mu(x)}$ and $U^{\mu(x)}=U_{x}$ since $U \cdot x$ is complex (Guillemin and Sternberg 1990). However, $\mu(x) \notin \mathfrak{p}$ and so $K_{x}$ does not coincide in general with $K^{\mu_{\mathfrak{p}}(x)}$.

If $M$ is a $U$-invariant compact connected complex submanifold of $(Z, \omega)$, then $v_{i \mathfrak{u}}$ constant is equivalent to $U$ being semisimple and $M=U / U_{\beta} \times \mu^{-1}(\beta)$. The above splitting is Riemannian (Gori and Podestà 2004) (see also Bedulli and Gori 2005; Biliotti 2009 for the same result under the assumption that $M$ is symplectic). In this paper we prove this splitting result without any assumption on $M$.

Theorem 2 Let $M$ be a $U^{\mathbb{C}}$-stable connected submanifold of a Kähler manifold $Z$ and let $\mu: M \longrightarrow \mathfrak{u}$ be the restricted momentum map. Then the norm-square of the momentum map $\|\mu\|^{2}$ is constant if and only if $U$ is semisimple and $M$ is $U$ equivariantly isometric to the product of a flag manifold and an embedded, closed submanifold which is acted on trivially by $U$.

Assume that $G$ is a real form of $U$. The momentum map of $U$ on $Z$ induces a gradient map $\mu_{i \mathfrak{k}}$ of $K^{\mathbb{C}}$ in $Z$. We say that $M$ is $G$-semistable if $M=\{p \in M$ : $\left.\overline{G \cdot p} \cap \mu_{\mathfrak{p}}^{-1}(0) \neq \emptyset\right\}$.

Theorem 3 Assume that $Z$ is compact and $U^{\mathbb{C}}$-semistable and $M$ is a $G$-semistable real connected submanifold of $Z$. Assume also $M$ is contained in the zero fiber of $\mu_{i \mathfrak{k}}$. Then the norm-square of the G-gradient map $\left\|\mu_{\mathfrak{p}}\right\|^{2}$ is constant if and only if $G$ is semisimple and $M$ is $K$-equivariantly isometric to the product of a real flag and an embedded closed submanifold which is acted on trivially by $K$.

\section{Closed Orbits and Gradient Map}

Let $(Z, \omega)$ be a Kähler manifold. Assume that $U^{\mathbb{C}}$ acts holomorphically on $Z$, that $U$ preserves $\omega$ and that there is a momentum map for the $U$ action on $Z$. Let $G \subset U^{\mathbb{C}}$ be a closed compatible subgroup and let $M$ be a $G$-invariant submanifold of $(Z, \omega)$ and let $\mu_{\mathfrak{p}}: M \longrightarrow \mathfrak{p}$ be the associated $G$-gradient map.

\section{Lemma 4 Let $x \in M$. Then:}

- if $x$ realizes a local maximum of $\nu_{\mathfrak{p}}$, then $G \cdot x=K \cdot x$ and so it is compact;

- if $G \cdot x$ is compact, then $G \cdot x=K \cdot x$ and $x$ is a critical point of $\nu_{\mathfrak{p}}$.

Proof If $x$ realizes a local maximum for $v_{\mathfrak{p}}$, then $v_{\mathfrak{p}}: G \cdot x \longrightarrow \mathbb{R}$ has a local maximum at $x$. By Corollary 6.12, p.21 in Heinzner et al. (2008), it follows $G \cdot x=K \cdot x$.

Assume $G \cdot x$ is compact. Then $v_{p}: G \cdot x \longrightarrow \mathbb{R}$ has a local maximum. Applying, again, Corollary 6.12 p.21 in Heinzner et al. (2008), we get $G \cdot x=K \cdot x$. We compute 
the differential of $v_{\mathfrak{p}}$ at $x$. It is easy to check

$$
\mathrm{d} v_{\mathfrak{p}}(v)=2\left\langle\left(\mathrm{~d} \mu_{\mathfrak{p}}\right)_{x}(v), \mu_{\mathfrak{p}}(x)\right\rangle
$$

Therefore, keeping in mind that $\operatorname{Ker}\left(\mathrm{d} \mu_{\mathfrak{p}}\right)_{x}=(\mathfrak{p} \cdot x)^{\perp}$, where $\mathfrak{p} \cdot x=\left\{\xi_{Z}(x): \xi \in \mathfrak{p}\right\}$ see Heinzner and Schwarz (2007), it follows $\left(d v_{p}\right)_{x}=0$ on $(\mathfrak{p} \cdot x)^{\perp}$. Since $G \cdot x=K \cdot x$, it follows $\mathfrak{p} \cdot x \subset \mathfrak{k} \cdot x$ and so, keeping in mind that $\nu_{\mathfrak{p}}$ is $K$-invariant, $\left(\mathrm{d} v_{p}\right)_{x}=0$ on $\mathfrak{p} \cdot x$ as well, proving $x$ is a critical point of $v_{\mathfrak{p}}$.

Lemma 5 Let $x \in M$ be such that $G \cdot x$ is compact. Let $\beta=\mu_{\mathfrak{p}}(x)$. Then

$$
\mathfrak{k} \cdot x=\mathfrak{p} \cdot x \stackrel{\perp}{\oplus} \mathfrak{k}^{\beta} \cdot x
$$

Therefore $\mathfrak{k} \cdot x=\mathfrak{p} \cdot x$ if and only if $\operatorname{dim} K \cdot x=\operatorname{dim} K \cdot \beta$.

Proof Since $G \cdot x$ is compact, by the above Lemma $G \cdot x=K \cdot x$. By the $K$-equivariance of $\mu_{\mathfrak{p}}$, it follows that $\mu_{\mathfrak{p}}: K \cdot x \longrightarrow K \cdot \beta$ is a smooth fibration. Therefore, keeping in mind that $\operatorname{Ker}\left(\mathrm{d} \mu_{\mathfrak{p}}\right)_{x}=(\mathfrak{p} \cdot x)^{\perp}$, we have

$$
(\mathfrak{p} \cdot x)^{\perp} \cap \mathfrak{k} \cdot x=\mathfrak{k}^{\beta} \cdot x
$$

Since $G \cdot x=K \cdot x$, we get

$$
\mathfrak{k} \cdot x=\mathfrak{p} \cdot x \stackrel{\perp}{\oplus}\left((\mathfrak{p} \cdot x)^{\perp} \cap \mathfrak{k} \cdot x\right)=\mathfrak{p} \cdot x \stackrel{\perp}{\oplus} \mathfrak{k}^{\beta} \cdot x .
$$

This also implies $\mathfrak{k} \cdot x=\mathfrak{p} \cdot x$ if and only if $\operatorname{dim} K \cdot x=\operatorname{dim} K \cdot \beta$, concluding the proof.

Assume that $G$ is a real form of $U^{\mathbb{C}}$. If $G \cdot x$ is compact then it is not in general true that $U^{\mathbb{C}} \cdot x$ is compact. Indeed, let $V$ be a complex vector space and let $\tau: G \longrightarrow$ $\operatorname{PGL}(V)$ be an irreducible faithful projective representation. Since the center of $G$ acts trivially, we may assume that $G$ is semisimple. The representation $\tau$ extends to an irreducible projective representation of $U^{\mathbb{C}}$. It is well-known that $U^{\mathbb{C}}$ has a unique closed orbit (Guillemin and Sternberg 1990). It is the orbit through a highest vector. On the other hand $G$ could have more than one closed orbit in $\mathbb{P}(V)$ Guivarc'h et al. (1998, Proposition 4.28, p. 58). The following result tells us that there exists a unique compact $G$-orbit contained in the unique compact orbit of $U^{\mathbb{C}}$.

Proposition 6 Let $M=U^{\mathbb{C}} \cdot x$ be a compact orbit. If $G$ is a real form of $U$, then there exists exactly one closed $G$-orbit in $M$.

Proof $U^{\mathbb{C}} \cdot x=U \cdot x$ and it is a flag manifold (Heinzner et al. 2008; Guillemin and Sternberg 1990). Applying a beautiful old Theorem of Wolf (1969), it follows that $G$ has a unique closed orbit in $M$. The $G$ orbit is given by the orbit throughout the maximum of the norm square of the gradient map (Heinzner et al. 2008).

The following result arises from Lemma 5. 
Corollary 7 Let $x \in M$ be such that $G \cdot x$ is compact. If $\operatorname{dim} K \cdot x=\operatorname{dim} K \cdot \mu_{\mathfrak{p}}(x)$, then $U^{\mathbb{C}} \cdot x$ is compact.

Proof Since $\mathfrak{u}=\mathfrak{k} \oplus i \mathfrak{p}$, it follows $\mathfrak{u} \cdot x=\mathfrak{k} \cdot x+i \mathfrak{p} \cdot x$. By Lemma $5, \mathfrak{k} \cdot x=\mathfrak{p} \cdot x$ and so $\mathfrak{u}^{\mathbb{C}} \cdot x=\mathfrak{u} \cdot x$. This implies $U \cdot x$ is open and closed in $U^{\mathbb{C}} \cdot x$. Therefore $U^{\mathbb{C}} \cdot x=U \cdot x$, concluding the proof.

The following result gives a necessary and sufficient condition so that $U^{\mathbb{C}} \cdot x$ is compact whenever $G \cdot x$ is.

Proposition 8 Let $x \in M$ be such that $G \cdot x$ is compact. If $G$ is a real form of $U^{\mathbb{C}}$, then $U^{\mathbb{C}} \cdot x$ is compact if and only if $i \mathfrak{k}^{\mu_{\mathfrak{p}}(x)} \cdot x \subseteq \mathfrak{u} \cdot x \cap i(\mathfrak{p} \cdot x)^{\perp}$. If $M$ is Lagrangian, then $U^{\mathbb{C}} \cdot x$ is compact if and only if $\mu_{\mathfrak{p}}: K \cdot x \longrightarrow K \cdot \mu_{\mathfrak{p}}(x)$ is a covering map. Moreover, $G \cdot x$ is a Lagrangian submanifold of $U^{\mathbb{C}} \cdot x$.

Proof Set $\beta=\mu_{\mathfrak{p}}(x)$. By Lemma $5, \mathfrak{k} \cdot x=\mathfrak{p} \cdot x \stackrel{\perp}{\oplus} \mathfrak{k}^{\beta} \cdot x$. Therefore, keeping in mind $\mathfrak{u}=\mathfrak{k} \oplus i \mathfrak{p}$, we have

$$
\mathfrak{u} \cdot x=\mathfrak{p} \cdot x \stackrel{\perp}{\oplus} \mathfrak{k}^{\beta} \cdot x+i \mathfrak{p} \cdot x .
$$

Since $i \mathfrak{k}^{\beta} \cdot x$ is orthogonal to $i \mathfrak{p} \cdot x$, it follows that $\mathfrak{u} \cdot x=\mathfrak{u}^{\mathbb{C}} \cdot x$, if and only if $i \mathfrak{k}^{\beta} \cdot x \subset \mathfrak{u} \cdot x \cap i(\mathfrak{p} \cdot x)^{\perp}$. If $M$ is Lagrangian, then $T_{x} Z=T_{x} M \stackrel{\perp}{\oplus} J\left(T_{x} M\right)$. Therefore

$$
\mathfrak{u} \cdot x=\mathfrak{p} \cdot x \stackrel{\perp}{\oplus} \mathfrak{k}^{\beta} \cdot x \stackrel{\perp}{\oplus} i \mathfrak{p} \cdot x .
$$

This implies $\mathfrak{u} \cdot x=\mathfrak{u}^{\mathbb{C}} \cdot x$ if and only if $i \mathfrak{k}^{\beta} \cdot x \subseteq i \mathfrak{p} \cdot x$. By the first part of the proof we get $U^{\mathbb{C}} \cdot x$ is compact if and only if $\mathfrak{k}^{\beta} \cdot x=\{0\}$ and so if and only if $\operatorname{dim} K \cdot x=\operatorname{dim} K \cdot \beta$. In particular $\mathfrak{p} \cdot x=\mathfrak{k} \cdot x$. This implies $\operatorname{dim}_{\mathbb{R}} G \cdot x=\operatorname{dim}_{\mathbb{C}} U^{\mathbb{C}} \cdot x$ and so $G \cdot x$ is a compact Lagrangian submanifold of $U^{\mathbb{C}} \cdot x$.

Proposition 9 Let $M$ be a $G$-invariant Lagrangian submanifold of $(Z, \omega)$. Let $x \in M$. Then $U^{\mathbb{C}} \cdot x$ is compact if and only if $\mathfrak{k} \cdot x=\mathfrak{p} \cdot x$. In particular $G \cdot x$ is compact and it is a Lagrangian submanifold of $U^{\mathbb{C}} \cdot x$.

Proof Since $M$ is Lagrangian, we have

$$
\mathfrak{u} \cdot x=\mathfrak{k} \cdot x \stackrel{\perp}{\oplus} i \mathfrak{p} \cdot x
$$

Therefore $\mathfrak{u} \cdot x=\mathfrak{u}^{\mathbb{C}} \cdot x$ if and only if $i \mathfrak{k} \cdot x \subseteq i \mathfrak{p} \cdot x$ and $\mathfrak{p} \cdot x \subseteq \mathfrak{k} \cdot x$ hence if and only if $\mathfrak{k} \cdot x=\mathfrak{p} \cdot x$. This also implies $G \cdot x$ is compact, $\operatorname{dim}_{\mathbb{R}} G \cdot \bar{x}=\operatorname{dim}_{\mathbb{C}} U^{\mathbb{C}} \cdot x$ and so $G \cdot x$ is a compact Lagrangian submanifold of $U^{\mathbb{C}} \cdot x$.

Proposition 10 Let $x \in Z$. Assume that both $G \cdot x$ and $U^{\mathbb{C}} \cdot x$ are compact. Then $\operatorname{dim}_{\mathbb{R}} U^{\mathbb{C}} \cdot x \leq 2 \operatorname{dim} G \cdot x$. If the equality holds then $G \cdot x$ is totally real.

Proof By Lemma $4 U^{\mathbb{C}} \cdot x=U \cdot x$ and $G \cdot x=K \cdot x$. Since $\mathfrak{u} \cdot x=\mathfrak{k} \cdot x+i \mathfrak{p} \cdot x$ and $\mathfrak{p} \cdot x \subseteq \mathfrak{k} \cdot x$, it follows that

$$
\operatorname{dim}_{\mathbb{R}} U^{\mathbb{C}} \cdot x \leq 2 \operatorname{dim} G \cdot x
$$


Note also that $\mathfrak{k}^{\mathbb{C}} \cdot x=\mathfrak{u}^{\mathbb{C}} \cdot x$. This implies $K^{\mathbb{C}} \cdot x$ is open in $U^{\mathbb{C}} \cdot x$. This remark is not new, see Heinzner et al. (2008), Heinzner and Stötzel (2006), and it arises from the Matsuki duality (Matsuki 1982). Finally, $2 \operatorname{dim} G \cdot x=\operatorname{dim}_{\mathbb{R}} U^{\mathbb{C}}$ if and only if $\mathfrak{k} \cdot x=\mathfrak{p} \cdot x$ and $\mathfrak{u} \cdot x=\mathfrak{k} \cdot x \oplus i \mathfrak{p} \cdot x$. In particular $G \cdot x$ is totally real in $U^{\mathbb{C}} \cdot x$.

The momentum map of $U$ on $Z$ induces a gradient map $\mu_{i \mathfrak{k}}$ of $K^{\mathbb{C}}$ in $Z$. Assume that $M$ is contained in the zero fiber of $\mu_{i \mathfrak{k}}$.

Proposition 11 Let $x \in M$. If $U^{\mathbb{C}} \cdot x$ is compact, then $G \cdot x$ is compact.

Proof Let $y \in U^{\mathbb{C}} \cdot x$. Since $\mu=\mu_{i \mathfrak{k}}+\mu_{\mathfrak{p}}, M \subseteq \mu_{i \mathfrak{k}}^{-1}(0)$ and keeping in mind that $U^{\mathbb{C}} \cdot x=U \cdot x$, it follows that

$$
\left\|\mu_{\mathfrak{p}}(y)\right\|^{2} \leq\|\mu(y)\|^{2}=\|\mu(x)\|^{2}=\left\|\mu_{\mathfrak{p}}(x)\right\|^{2} .
$$

Hence $v_{\mathfrak{p}}: U^{\mathbb{C}} \cdot x \longrightarrow \mathbb{R}$ achieves its maximum in $x$. By Lemma $4, G \cdot x$ is compact.

We say that $M$ is $G$-semistable if $M=\left\{p \in M: \overline{U^{\mathbb{C}} \cdot p} \cap \mu_{\mathfrak{p}}^{-1}(0) \neq \emptyset\right\}$. In the papers (Heinzner and Schwarz 2007; Heinzner et al. 2008), the authors proved if $M$ is $G$-semistable then $G \cdot x$ is closed if and only if $G \cdot x \cap \mu_{\mathfrak{p}}^{-1}(0) \neq \emptyset$. As an application we get the following result.

Proposition 12 Assume that $(Z, \omega)$ is $U^{\mathbb{C}}$-semistable and $M$ is $G$-semistable, it is contained in the zero fiber of $\mu_{i \mathfrak{k}}$. Let $x \in M$. If $G \cdot x$ is closed, then $U^{\mathbb{C}} \cdot x$ is closed as well.

Proof If $G \cdot x$ is closed then $G \cdot x \cap \mu_{\mathfrak{p}}^{-1}(0) \neq \emptyset$. Since $\mu_{\mathfrak{p}}^{-1}(0) \cap M=\mu^{-1}(0) \cap M$, it follows that $U^{\mathbb{C}} \cdot x \cap \mu^{-1}(0) \neq \varnothing$ as well and so $U^{\mathbb{C}} \cdot x$ is closed.

A corollary we prove a well-known result of Birkes (1971), see also Heinzner and Stötzel (1962).

Corollary 13 Let $G$ be a real form of $U$. Let $V$ be complex vector space and $W$ be real subspace of $V$ such that $V=W^{\mathbb{C}}$. Assume that $G$ acts on $W$. Let $w \in W$. Then $G \cdot w$ is closed if and only if $U^{\mathbb{C}} \cdot x$ is closed.

Proof It is well-known that $V$, respectively $W$, is $U^{\mathbb{C}}$-semistable, respectively $G$ semistable (Richardson and Slodowoy 1990), see also Biliotti (2021). Since $W$ is a Lagrangian subspace of $V$, applying the above Proposition it follows that $G \cdot x$ closed implies $U^{\mathbb{C}} \cdot x$ is closed as well. Vice-versa, assume that $U^{\mathbb{C}} \cdot x$ is closed. The $G$-action on $V$ is holomorphic and $W$ is a real form of $V$. Since $G$ is a real form of $U^{\mathbb{C}}$ the dimension of any $G$-orbit in $U^{\mathbb{C}} \cdot x \cap W$ coincides with the real dimension of $U^{\mathbb{C}} \cdot x$ (Borel and Harish-Chandra 1962, Proposition 2.3). Then all these orbits, and so $G \cdot x$, are closed. 


\section{Norm Square of the Gradient Map}

We investigate splitting results for $G$-invariant real submanifolds of $(Z, \omega)$.

Proposition 14 Let $M$ be a $G$-stable connected submanifold of $Z$ and let $\mu_{\mathfrak{p}}: M \longrightarrow$ $\mathfrak{p}$ be the restricted gradient map. Then the norm-square of the gradient map $\nu_{\mathfrak{p}}$ : $M \longrightarrow \mathbb{R}$ is constant if and only if any $G$-orbit is compact.

Proof Assume $v_{p}$ is constant. Let $x \in M$. Then $v_{\mathfrak{p}}: G \cdot x \longrightarrow \mathbb{R}$ is constant and so $v_{\mathfrak{p}}$ has a maximum on $x$. By Lemma $4 G \cdot x=K \cdot x$ and so it is compact. Vice-versa, assume that any $G$-orbit is compact. By Lemma $4\left(\mathrm{~d} v_{p}\right)_{x}=0$ for any $x \in M$. Since $M$ is connected it follows $v_{\mathfrak{p}}$ is constant.

The following result is proved in Heinzner et al. (2008). For the sake of completeness we give a proof.

Proposition 15 Let $M$ be a $G$-stable connected submanifold of $Z$ and let $\mu_{\mathfrak{p}}: M \longrightarrow$ $\mathfrak{p}$ be the restricted gradient map. If $\nu_{\mathfrak{p}}$ is constant, then $\mu_{\mathfrak{p}}(M)=K \cdot \beta, \mu^{-1}(\beta)$ is a submanifold and the following splitting

$$
M=K \times{ }_{K}^{\beta} \mu_{\mathfrak{p}}^{-1}(\beta),
$$

holds.

Proof Since $v_{\mathfrak{p}}$ is constant, it follows that $M=S_{\beta}$, where $S_{\beta}$ is the maximal strata, and $\mu_{\mathfrak{p}}\left(S_{\beta}\right)=\mu_{\mathfrak{p}}(M)=K \cdot \beta$ (Heinzner et al. (2008, p. 21). In particular $M=K \mu_{\mathfrak{p}}^{-1}(\beta)$ and we may think $\mu_{\mathfrak{p}}: M \longrightarrow K \cdot \beta$. Therefore $\beta$ is a regular value and so $\mu_{\mathfrak{p}}^{-1}(\beta)$ is a $K^{\beta}$-invariant submanifold of $M$.

Let $x \in \mu_{\mathfrak{p}}^{-1}(\beta)$. By the $K$-equivariance of $\mu_{\mathfrak{p}}$, it is easy to check $K \cdot x \cap \mu_{\mathfrak{p}}^{-1}(\beta)=$ $K^{\beta} \cdot x$. We claim that the same holds infinitesimally, i.e., $T_{x} \mu_{\mathfrak{p}}^{-1}(\beta) \cap \mathfrak{k} \cdot x=\mathfrak{k}^{\beta} \cdot x$. Indeed, let $v \in T_{x} \mu_{\mathfrak{p}}^{-1}(\beta) \cap \mathfrak{k} \cdot x$. Let $\xi \in \mathfrak{k}$ such that $v=\xi_{M}(x)$. Since $T_{x} \mu_{\mathfrak{p}}^{-1}(\beta)=$ $\operatorname{Ker}\left(\mathrm{d} \mu_{\mathfrak{p}}\right)_{x}$, we get

$$
0=\left.\frac{\mathrm{d}}{\mathrm{dt}}\right|_{t=0} \mu_{\mathfrak{p}}(\exp (t \xi) x)=\left.\frac{\mathrm{d}}{\mathrm{dt}}\right|_{t=0} \operatorname{Ad}(\exp (t \xi)) \beta
$$

and so $v \in \mathfrak{k}^{\beta} \cdot x$.

We define the map

$$
\Psi: K \times_{K^{\beta}} \mu_{\mathfrak{p}}^{-1}(\beta) \longrightarrow M \quad[k, x] \mapsto k x .
$$

It is easy to check that $\Psi$ is $K$-equivariant and smooth. Since $\mu_{\mathfrak{p}}(M)=K \cdot \beta$ it follows $M=K \cdot \mu_{\mathfrak{p}}^{-1}(\beta)$ and so $\Psi$ is surjective. It is also injective since $k x=k^{\prime} x$ if and only if $k^{\prime-1} k \in K^{\beta}$, proving it is bijective. Now, we proof that $\Psi$ is a local diffeomorphism. This implies that $\Psi$ is a diffeomorphism concluding the proof. Note that it is enough to prove $\mathrm{d} \Psi_{[e, x]}$ is a diffeomorphism by the $K$-equivariance. Now,

$$
T_{x} M=(\mathfrak{p} \cdot x) \oplus(\mathfrak{p} \cdot x)^{\perp}=(\mathfrak{p} \cdot x) \oplus T_{x} \mu_{\mathfrak{p}}^{-1}(\beta) .
$$


By Proposition 14 any $G$ orbit is a $K$ orbit. This implies $\mathfrak{p} \cdot x \subset \mathfrak{k} \cdot x$. Since $\mathfrak{k}^{\beta} \cdot x \subset$ $(\mathfrak{p} \cdot x)^{\perp}$, it follows that the map

$$
\mathfrak{p} \cdot x \hookrightarrow \mathfrak{k} \cdot x \longrightarrow \mathfrak{k} \cdot x / \mathfrak{k}^{\beta} \cdot x,
$$

is injective. Therefore $\mathrm{d} \Psi_{[e, x]}$ is surjective. Since $\Psi$ is bijective it follows that $\mathrm{d} \Psi_{[e, x]}$ must be bijective.

We are ready to prove the splitting results.

Proof of Theorem 2 Since $v$ is constant, applying Proposition 14 it follows that any $U^{\mathbb{C}}$-orbit is compact and it is a complex $U$ orbit. Then for any $x \in M$, we have $U_{x}=U_{\mu(x)}$ (Guillemin and Sternberg 1990). Since $U_{\mu(x)}$ is a centralizer of a torus, then the center of $U$ does not act on $M$ and so $U$ is semisimple. By the above proposition $M=U / U^{\beta} \times \mu^{-1}(\beta)$ and for every $x \in \mu^{-1}(\beta), U_{x}=U^{\beta}$ and so $U_{x}$ acts trivially on $\mu^{-1}(\beta)$. If $x \in \mu^{-1}(\beta)$, then

$$
T_{x} M=(i \mathfrak{u} \cdot x) \stackrel{\perp}{\oplus} T_{x} \mu^{-1}(\beta)=T_{x} U \cdot x \stackrel{\perp}{\oplus} T_{x} \mu^{-1}(\beta) .
$$

This implies that the $U$-action on $M$ is polar with section $\mu^{-1}(\beta)$ (Dadok 1985) and so $\mu^{-1}(\beta)$ is totally geodesic. We claim that the above splitting is Riemannian.

Let $\xi \in \mathfrak{u}$ and let $\xi_{M}$ the induced vector field. It is enough to prove that the function $g\left(\xi_{M}, \xi_{M}\right)$ is constant when restricted to $\mu^{-1}(\beta)$.

Let $x \in \mu^{-1}(p)$ and $v \in T_{x} \mu^{-1}(p)$. We may extend $v$ to a vector field on a neighborhood of $p$, that we denote by $X$, such that $g\left(X, \xi_{M}\right)=0$ for any $z \in W$ and for any $\xi \in \mathfrak{u}$. Indeed, let $\xi_{1}, \ldots, \xi_{k} \in \mathfrak{u}$ such that $\left(\xi_{1}\right)_{M}(x), \ldots\left(\xi_{k}\right)_{M}(x)$ is a basis of $T_{x} U \cdot x$. Since the $U$ action on $M$ has only one type of orbit, it follows that there exists a neighborhood $W$ of $x$ such that $\left(\xi_{1}\right)_{M}(y), \ldots,\left(\xi_{k}\right)_{M}(y)$ is a basis of $T_{y} U \cdot y$ for any $y \in W$. Applying a Gram-Schmidt process we get an orthonormal basis $\left\{Y_{1}, \ldots, Y_{k}\right\}$ of $T_{y} U \cdot y$ for any $y \in W$. Let $\tilde{X}$ any local extension of $v$. Then

$$
X=\tilde{X}-g\left(Y_{1}, \tilde{X}\right) Y_{1}-\cdots-g\left(Y_{k}, \tilde{X}\right) Y_{k}
$$

satisfies the above conditions. Moreover, for any $z \in \mu_{\mathfrak{p}}^{-1}(\beta) \cap W$, the vector field $X$ lies in $T_{z} \mu_{\mathfrak{p}}^{-1}(\beta)$ due to the orthogonal splitting $T_{z} M=T_{z} U \cdot z \stackrel{\perp}{\oplus} T_{z} \mu_{\mathfrak{p}}^{-1}(\beta)$.

Let $v_{M}=-J\left(\xi_{M}\right)$ Then $J\left(v_{M}\right)=\xi_{M}$. Since $M=U / U_{\beta} \times \mu^{-1}(p)$, it follows $\left[X, \xi_{M}\right]=\left[X, v_{M}\right]=0$ along $\mu_{\mathfrak{p}}^{-1}(\beta)$. By the closedness of $\omega$, we have

$$
\mathrm{d} \omega\left(v, v_{M}(x), \xi_{M}(x)\right)=0
$$

On the other hand, by the expression of the differential (Kobayashi and Nomizu 1996), we have

$$
\begin{aligned}
\mathrm{d} \omega\left(v, v_{M}(x), \xi_{M}(x)\right)= & X \omega\left(v_{M}, \xi_{M}\right)+v_{M} \omega\left(\xi_{M}, X\right)+\xi_{M} \omega\left(X, v_{M}\right) \\
& -\omega\left(\left[X, v_{M}\right], \xi_{M}\right)-\omega\left(\left[v_{M}, \xi_{N}\right], X\right)-\omega\left(\left[\xi_{M}, X\right], Y\right) .
\end{aligned}
$$


Now, $\omega\left(\left[X, v_{M}\right], \xi_{M}\right)=\omega\left(\left[\xi_{M}, X\right], Y\right)=0$ due to the fact that $\left[X, v_{M}\right](x)=$ $\left[\xi_{M}, X\right](x)=0$, The term $\omega\left(\left[v_{M}, \xi_{N}\right], X\right)=0$, since

$$
\omega\left(\left[v_{M}, \xi_{N}\right], X\right)=g\left(J\left(\left[v_{M}, \xi_{M}\right], X\right)=0\right.
$$

due to the facts that any $U$-orbit is complex and the splitting $T_{x} M=T_{x} \mu^{-1}(\beta) \stackrel{\perp}{\oplus}$ $T_{x} U \cdot x$ holds. Finally, $v_{M} \omega\left(\xi_{M}, X\right)=0$, respectively $\xi_{M} \omega\left(X, v_{M}\right)=0$, due to the fact that

$$
\omega\left(\xi_{M}, X\right)=g\left(J \xi_{M}, X\right)=0
$$

respectively,

$$
\omega\left(X, v_{M}\right)=g\left(J X, v_{M}\right)=-g\left(X, J v_{M}\right)=0,
$$

along $U \cdot x$. Therefore

$$
0=\mathrm{d} \omega\left(v, v_{M}(x), \xi_{M}(x)\right)=X \omega\left(v_{M}, \xi_{M}\right)=X g\left(J\left(v_{M}\right), \xi_{M}\right)=X g\left(\xi_{M}, \xi_{M}\right),
$$

and so $g\left(\xi_{M}, \xi_{M}\right)$ is constant along $\mu_{\mathfrak{p}}^{-1}(\beta)$ and the result is proved.

Proof of Theorem 3 By Proposition $15 M=K \times_{K^{\beta}} \mu_{\mathfrak{p}}^{-1}(\beta)$. By Proposition 14 it follows $G \cdot x$ is compact for any $x \in \mu_{\mathfrak{p}}^{-1}(\beta)$. Let $x \in \mu_{\mathfrak{p}}^{-1}(\beta)$. By Proposition 12, $U^{\mathbb{C}} \cdot x$ is compact as well. Since $M$ is contained in the zero fiber of $\mu_{i \mathfrak{k}}$, it follows that $\mu_{\mathfrak{p}}(x)=\mu(x)=\beta$. This implies $K_{x}=K \cap U_{x}=K \cap U^{\beta}=K^{\beta}$ for any $x \in \mu_{\mathfrak{p}}^{-1}(\beta)$ and so $M=K / K^{\beta} \times \mu_{\mathfrak{p}}^{-1}(\beta)$. The Lie algebra of the center of $G$ is contained in the Lie algebra of the center of $U^{\mathbb{C}}$. On the other hand, the Lie algebra of the center of $U^{\mathbb{C}}$ is the complexification of the Lie algebra of the center of $U$ which acts trivially on $M$. This implies $G$ is semisimple. Finally, keeping in mind that $\omega$ is closed and $U^{\mathbb{C}} \cdot x$ is compact for any $x \in \mu_{\mathfrak{p}}^{-1}(\beta)$, applying the same idea of the above proof we get the splitting $M=K / K^{\beta} \times \mu_{\mathfrak{p}}^{-1}(\beta)$ is Riemannian.

Acknowledgements The author would like to thank the anonymous referee for helpful comments.

Funding Open access funding provided by Universitá degli Studi di Parma within the CRUI-CARE Agreement.

Open Access This article is licensed under a Creative Commons Attribution 4.0 International License, which permits use, sharing, adaptation, distribution and reproduction in any medium or format, as long as you give appropriate credit to the original author(s) and the source, provide a link to the Creative Commons licence, and indicate if changes were made. The images or other third party material in this article are included in the article's Creative Commons licence, unless indicated otherwise in a credit line to the material. If material is not included in the article's Creative Commons licence and your intended use is not permitted by statutory regulation or exceeds the permitted use, you will need to obtain permission directly from the copyright holder. To view a copy of this licence, visit http://creativecommons.org/licenses/by/4.0/.

\section{References}

Bedulli, L., Gori, A.: A splitting result for compact symplectic manifolds. Results Math. 47, 194-198 (2005) 
Biliotti, L.: A note on moment map on symplectic manifolds. Bull. Belg. Math. Soc. Simon Stevin 16, 107-116 (2009)

Biliotti, L.: The Kempf-Ness Theorem and invariant theory for real reductive representations. Sao Paulo J. Math. Sci. 15(1), 54-74 (2021)

Birkes, D.: Orbits of linear algebraic groups. Ann. Math. 93(2), 459-475 (1971)

Borel, A., Harish-Chandra: Arithmetic subgroups of algebraic groups. Ann. Math. 75(2), 485-535 (1962)

Dadok, J.: Polar coordinates induced by actions of compact Lie groups. Trans. Am. Math. Soc. 288(1), 125-137 (1985)

Gori, A., Podestà, F.: A note on the moment map on compact Kähler manifold. Ann. Global. Anal. Geom. 26, 315-318 (2004)

Guillemin, V., Sternberg, S.: Symplectic Techniques in Physics, 2nd edn. Cambridge University Press, Cambridge (1990)

Guivarc'h, Y., Ji, L., Taylor, J.C.: Compactifications of symmetric spaces. Progress in Mathematics, vol. 156. Birkhäuser Boston Inc., Boston (1998)

Heinzner, P., Schwarz, G.W.: Cartan decomposition of the moment map. Math. Ann. 337(1), 197-232 (2007)

Heinzner, P., Stötzel, H.: Critical Points of the Square of the Momentum Map, Global Aspects of Complex Geometry, pp. 211-226. Springer, Berlin (2006)

Heinzner, P., Schwarz, G.W., Stötzel, H.: Stratifications with respect to actions of real reductive groups. Compos. Math. 144(1), 163-185 (2008)

Helgason, S.: Differential Geometry, Lie Groups and Symmetric Spaces, Corrected reprint of the 1978 original. Graduate Studies in Mathematics, 34. American Mathematical Society, Providence, RI (2001)

Kirwan, F.C.: Cohomology of Quotients in Symplectic and Algebraic Geometry. Mathematical Notes, vol. 31. Princeton University Press, Princeton (1984)

Knapp, A.W.: Lie Groups Beyond an Introduction. Progress in Mathematics, vol. 140, 2nd edn. Birkhäuser Boston Inc., Boston (2002)

Kobayashi, S., Nomizu, K.: Foundations of Differential Geometry, vol. I. Wiley, New York (1996)

Matsuki, T.: Orbits on affine symmetric spaces under the action of parabolic subgroups. Hiroshima Math. J. 12, 307-320 (1982)

Richardson, R.W., Slodowoy, P.J.: Minumun vectors for real reductive algebraic groups. J. Lond. Math. Soc. 42(2), 409-429 (1990)

Wolf, J.: The action of a real semisimple group on a complex flag manifold I. Orbit structure and holomorphic arc. Bull. Am. Math. Soc. 75, 1121-1237 (1969)

Publisher's Note Springer Nature remains neutral with regard to jurisdictional claims in published maps and institutional affiliations. 